\title{
Front Matter: Volume 9363
}

, "Front Matter: Volume 9363," Proc. SPIE 9363, Gallium Nitride Materials and Devices X, 936301 (6 May 2015); doi: 10.1117/12.2193017

SPIE. Event: SPIE OPTO, 2015, San Francisco, California, United States 


\section{PROCEEDINGS OF SPIE}

\section{Gallium Nitride Materials and Devices $X$}

Jen-Inn Chyi

Hiroshi Fujioka

Hadis Morkoç

Editors

9-12 February 2015

San Francisco, California, United States

Sponsored and Published by

SPIE

Volume 9363

Proceedings of SPIE 0277-786X, V. 9363

SPIE is an international society advancing an interdisciplinary approach to the science and application of light.

Gallium Nitride Materials and Devices X, edited by Jen-Inn Chyi, Hiroshi Fujioka,

Hadis Morkoç, Proc. of SPIE Vol. 9363, 936301 · (C) 2015 SPIE

CCC code: $0277-786 X / 15 / \$ 18 \cdot$ doi: $10.1117 / 12.2193017$

Proc. of SPIE Vol. $9363936301-1$

Downloaded From: https://www.spiedigitallibrary.org/conference-proceedings-of-spie on 26 Apr 2023 Terms of Use: https://www.spiedigitallibrary.org/terms-of-use 
The papers included in this volume were part of the technical conference cited on the cover and title page. Papers were selected and subject to review by the editors and conference program committee. Some conference presentations may not be available for publication. The papers published in these proceedings reflect the work and thoughts of the authors and are published herein as submitted. The publisher is not responsible for the validity of the information or for any outcomes resulting from reliance thereon.

Please use the following format to cite material from this book:

Author(s), "Title of Paper," in Gallium Nitride Materials and Devices X, edited by Jen-Inn Chyi, Hiroshi Fujioka, Hadis Morkoç, Proceedings of SPIE Vol. 9363 (SPIE, Bellingham, WA, 2015) Article CID Number.

ISSN: 0277-786X

ISBN: 9781628414530

Published by

SPIE

P.O. Box 10, Bellingham, Washington 98227-0010 USA

Telephone +1 3606763290 (Pacific Time) · Fax +1 3606471445

SPIE.org

Copyright (c) 2015, Society of Photo-Optical Instrumentation Engineers.

Copying of material in this book for internal or personal use, or for the internal or personal use of specific clients, beyond the fair use provisions granted by the U.S. Copyright Law is authorized by SPIE subject to payment of copying fees. The Transactional Reporting Service base fee for this volume is $\$ 18.00$ per article (or portion thereof), which should be paid directly to the Copyright Clearance Center (CCC), 222 Rosewood Drive, Danvers, MA 01923. Payment may also be made electronically through CCC Online at copyright.com. Other copying for republication, resale, advertising or promotion, or any form of systematic or multiple reproduction of any material in this book is prohibited except with permission in writing from the publisher. The CCC fee code is 0277-786X/15/\$18.00.

Printed in the United States of America.

Publication of record for individual papers is online in the SPIE Digital Library.

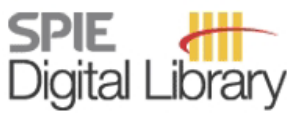

SPIEDigitalLibrary.org

Paper Numbering: Proceedings of SPIE follow an e-First publication model, with papers published first online and then in print. Papers are published as they are submitted and meet publication criteria. A unique citation identifier (CID) number is assigned to each article at the time of the first publication. Utilization of CIDs allows articles to be fully citable as soon as they are published online, and connects the same identifier to all online, print, and electronic versions of the publication. SPIE uses a six-digit CID article numbering system in which:

- The first four digits correspond to the SPIE volume number.

- The last two digits indicate publication order within the volume using a Base 36 numbering

system employing both numerals and letters. These two-number sets start with 00, 01, 02, 03, 04, $05,06,07,08,09,0 A, 0 B \ldots$. OZ, followed by 10-1Z, 20-2Z, etc.

The CID Number appears on each page of the manuscript. The complete citation is used on the first page, and an abbreviated version on subsequent pages. 


\title{
Contents
}

\author{
vii Authors \\ xi Conference Committee
}

GROWTH I

936302 Ammonothermal growth of polar and non-polar bulk GaN crystal (Invited Paper) [9363-1]

GROWTH III

9363 OE HVPE growth of AlxGa $\mathbf{a}_{1-x N}$ templates for UV-LED applications (Invited Paper) [9363-13]

9363 OF Homoepitaxial HVPE GaN growth on non- and semi-polar seeds [9363-14]

\section{MATERIAL CHARACTERIZATION I}

$93630 \mathrm{O}$ Optical properties and band structure of highly doped gallium nitride (Invited Paper) [9363-15]

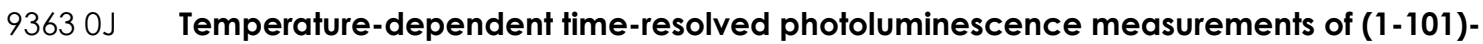
oriented semi-polar AIGaN/GaN MQWs [9363-18]

9363 OK Photoluminescence behavior of amber light emitting GalnN-GaN heterostructures [9363-19]

$9363 \mathrm{OL}$ Identification of point defects in HVPE-grown GaN by steady-state and time-resolved photoluminescence [9363-20]

9363 OM Study of interface state trap density on characteristics of MOS-HEMT [9363-21]

\section{MATERIAL CHARACTERIZATION II}

$9363 \mathrm{OP}$ Stress related aspects of GaN technology physics [9363-24]

\section{NANOSTRUCTURES AND DEVICES I}

9363 OT Photocathode electron beam sources using GaN and InGaN with NEA surface (Invited Paper) [9363-28]

9363 OU Ordered arrays of InGaN/GaN dot-in-a-wire nanostructures as single photon emitters (Invited Paper) [9363-29] 
$9363 \mathrm{OZ}$ Optical properties of small GaN-Al $0.5 \mathrm{Ga}_{0.5} \mathrm{~N}$ quantum dots grown on (11-22) GaN templates [9363-34]

\section{ELECTRON DEVICES I}

936310 Present and future of GaN power devices and their applications (Invited Paper) [9363-35]

936311 Direct comparison of GaN-based e-mode architectures (recessed MISHEMT and p-GaN HEMTs) processed on 200mm GaN-on-Si with Au-free technology (Invited Paper) [9363-36]

936312 Enhancement of AIGaN/GaN high-electron mobility transistor off-state drain breakdown voltage via backside proton irradiation (Invited Paper) [9363-37]

936314 Trapping processes related to iron and carbon doping in AIGaN/GaN power HEMTs [9363-39]

\section{LASER DIODES I}

936318 InGaN power laser chips in a novel 50W multi-die package (Invited Paper) [9363-43]

$93631 \mathrm{~A}$ Advances in single mode and high power AIGalnN laser diode technology for systems applications [9363-45]

9363 ID Ultralow threshold electrically injected AIGaN nanowire ultraviolet lasers on Si [9363-48]

\section{LASER DIODES II}

$93631 \mathrm{G}$ Status and future of GaN-based vertical-cavity surface-emitting lasers (Invited Paper) [9363-51]

LEDS I

9363 1K High-power UV-B LEDs with long lifetime (Invited Paper) [9363-55]

$93631 \mathrm{~L} \quad$ Progress of high-power deep-ultraviolet LEDs (Invited Paper) [9363-56]

9363 1M High-efficiency UV LEDs on sapphire (Invited Paper) [9363-57]

$93631 \mathrm{P}$ Current spreading in UV-C LEDs emitting at $235 \mathrm{~nm}$ [9363-60] 
$93631 Q \quad$ Study of efficiency droop in InGaN/GaN light emitting diodes with V-shape pits (Invited Paper) [9363-61]

9363 IR Efficiency droop in nitride LEDs revisited: impact of excitonic recombination processes [9363-62]

9363 is Bipolar Monte Carlo simulation of electrons and holes in III-N LEDs [9363-63]

LEDS III

9363 1T Semi/non-polar nitride quantum wells for high-efficient light emitters (Invited Paper) [9363-64]

$93631 \mathrm{U}$ Spatial variations of optical properties of semipolar InGaN quantum wells [9363-65]

$93631 \mathrm{~V}$ Investigation of facet-dependent InGaN growth for core-shell LEDs [9363-66]

LEDS IV

$93631 \mathrm{InGaN}$ LEDs prepared on $\boldsymbol{\beta}-\mathrm{Ga}_{2} \mathrm{O}_{3}$ (-201) substrates (Invited Paper) [9363-71]

POSTER SESSION

936325 Deep level transient spectroscopy on light-emitting diodes based on (In,Ga)N/GaN nanowire ensembles [9363-77]

936328 X-ray diffraction study of A- plane non-polar InN epilayer grown by MOCVD [9363-80]

9363 2A Vertical excitation profile in diffusion injected multi-quantum well light emitting diode structure [9363-82]

$93632 \mathrm{~B} \quad$ Numerical analysis on the effect of electron blocking layer in 365-nm ultraviolet lightemitting diodes [9363-83]

$93632 \mathrm{H} \quad$ Nonradiative recombination mechanisms in InGaN/GaN light-emitting diodes analyzed by various device characterization techniques [9363-89]

$936321 \quad$ Enhancement of optical and structural quality of semipolar (11-22) GaN by introducing nanoporous SiN interlayers [9363-91]

9363 2J Enhancement of coherent acoustic phonons in InGaN multiple quantum wells [9363-92]

$93632 \mathrm{~N} \quad$ Strong carrier localization in stacking faults in semipolar (11-22) GaN [9363-96]

936320 Strong exciton-photon coupling in hybrid InGaN-based microcavities on GaN substrates [9363-97] 
9363 2P Indium-incorporation efficiency in semipolar (11-22) oriented InGaN-based light emitting diodes [9363-98]

$93632 \mathrm{U}$ Active region dimensionality and quantum efficiencies of InGaN LEDs from temperature dependent photoluminescence transients [9363-103] 


\title{
Authors
}

Numbers in the index correspond to the last two digits of the six-digit citation identifier (CID) article numbering system used in Proceedings of SPIE. The first four digits reflect the volume number. Base 36 numbering is employed for the last two digits and indicates the order of articles within the volume. Numbers start with 00, 01, 02, 03, 04, 05, 06, 07, 08, 09, OA, OB...0Z, followed by 10-12, 20-2Z, etc.

\author{
Allsopp, Duncan W.E., 1V \\ Amano, H., OT \\ Amilusik, M., OF \\ Asada, K., $1 \mathrm{~L}$ \\ Avramescu, Adrian, 18 \\ Avrutin, Vitaliy, 0J, 2I, 2J, 2N, 2O, 2P, $2 \mathrm{U}$ \\ Bahat-Treidel, E., 14 \\ Bertram, Frank, OU, 2I, 2O, 2P \\ Bilenko, Yuri, $1 \mathrm{M}$ \\ Bisi, D., 14 \\ Boćkowski, Michal, OF, IA \\ Brault, Julien, $0 Z$ \\ Bremers, H., IR \\ Briot, Olivier, 28 \\ Calleja, Enrique, ou \\ Calleja, José M., OU \\ Can, Nuri, 2I, 2P, 2U \\ Cassabois, Guillaume, $\mathrm{OZ}$ \\ Chang, Chiao-Yun, 1Q \\ Chang, Jih-Yuan, 2B \\ Chen, Fang-Ming, 2B \\ Chernysheva, Ekaterina, OU \\ Christen, Juergen, 0U, 2I, 2O, 2P \\ Czernecki, Robert, 1A \\ Damilano, Benjamin, $\mathrm{OK}, \mathrm{OZ}$ \\ Das, Saikat, 2I, 2N, 2P \\ De Jaeger, Brice, 11 \\ de Mierry, Philippe, OK, OZ \\ De Santi, C., 14, 25 \\ Decoutere, Stefaan, 11 \\ Demchenko, D. O., OL \\ DenBaars, Steven P., $1 \mathrm{U}$ \\ Dobbertin, Thomas, 18 \\ Dobrinsky, Alex, 1M \\ Eberspach, Florian, $1 \mathrm{P}$ \\ Edwards, Paul R., 1V \\ Eichler, Christoph, 18 \\ Einfeldt, Sven, 1K, 1P \\ Enslin, Johannes, $1 \mathrm{~K}$ \\ Feezell, Daniel F., $1 G$ \\ Feneberg, Martin, OG \\ Fijalkowski, M., OF \\ Franke, Alexander, 20 \\ Fujioka, A., 1L \\ Fujisawa, Hideo, 02 \\ Funato, Mitsuru, $1 T$ \\ Gačević, Žarko, OU \\ García-Lepetit, Noemi, OU \\ Gaska, Remis, $1 M$
}

Geelhaar, L., 25

Gelžinyte, Kristina, $1 \mathrm{U}$

Gerhard, M., IR

Gerhard, Sven, 18

Gil, Bernard, OJ, OK, 0Z, 28

Girgel, lonut, 1V

Glaab, Johannes, 1K, IP

Goldhahn, Rüdiger, OG

González Calbet, José M., OU

Grzanka, E., OF

Grzegory, I., OF

Guillet, Thierry, 0 Z

Guttmann, Martin, 1K, 1P

Hafiz, Shopan D., 2J

Hager, Thomas, 18

Han, Dong-Pyo, $2 \mathrm{H}$

Hangleiter, A., IR

Helava, H., OL

Hilt, O., 14

Honda, Y., OT

Horn, Markus, 18

Horng, Ray-Hua, OE, OM

Hsu, Ta-Cheng, 12

Hung, Ming-Hsien, OM

Hwang, Y.-H., 12

lizuka, Kazuyuki, 1 Z

Ishinabe, Takayuki, 02

Ivanov, Ruslan, $1 \mathrm{U}$

Iwinska, M., OF

Izyumskaya, Natalia, 0J, 2I, 2N, 2P

Jain, Rakesh, $1 \mathrm{M}$

Kagamitani, Yuji, 02

Kalincev, D., IR

Kang, Xuanwu, 11

Kawabata, Shinichiro, 02

Kawakami, Yoichi, IT

Kelly, Antony E., 1A

Khachapuridze, P. A., OF

Kim, Hyun-Sung, $2 \mathrm{H}$

Kim, Kyu-Sang, $2 \mathrm{H}$

Kishikawa, D., $1 \mathrm{~L}$

Kivisaari, Pyry, 1S, 2A

Knaver, Arne, IP

Kneissl, Michael, 1K, 1P

Koch, M., IR

Koenig, Harald, 18

Kojima, Atsuhiko, 02

Kolbe, Tim, 1K

Kosugi, T., $1 \mathrm{~L}$ 
Krupczynska, P., OF

Kruse, A., 1R

Kucharski, Robert, 1A

Kuhn, Christian, 1K, IP

Kuo, Hao-Chung, 2B

Kuo, Yen-Kuang, 2B

Kuramata, Akito, $1 Z$

Lange, Karsten, OG

Langer, T., IR

Lapeyrade, Mickael, 1K, IP

Law, Mark E., 12

Lazić, Snežana, oU

Le Boulbar, Emmanuel, $1 \mathrm{~V}$

Lekhal, Kaddour, OK

Leszczyński, Mike, 1A

Li, Heng, 1Q

Li, K. H., ID

Liang, Jia-Hao, $\mathrm{OE}$

Lin, Bing-Cheng, 2B

Liu, Gavin, $1 Z$

Liv, X., 1D

Lobo-Ploch, Neysha, 1K, IP

Loeffler, Andreas, 18

Lu, Tien-Chang, $1 Q$

Lucznik, B., OF

Maekawa, T., OT

Makarov, Yu., OL

Marcinkevičius, Saulius, $1 \mathrm{U}$

Marcon, Denis, 11

Marona, Lujca, 1A

Martin, Robert W., IV

Massies, Jean, $0 Z$

Meguro, T., OT

Mehnke, Frank, 1K, IP

Meneghesso, G., 14

Meneghini, M., 14, 25

Metzner, Sebastian, 2I, 2P

Mi, Z., ID

Mikawa, Yutaka, 02

Mochizuki, Tae, 02

Mogilatenko, Anna, 1K

Monavarian, Morteza, 0J, 2I, 2J, 2N, 2P, 2U

Moret, Matthieu, 28

Morishima, Yoshikatsu, $1 \mathrm{Z}$

Morkoç, Hadis, 0J, 2I, 2J, 2N, 2O, 2P, 2U

Mueller, Jens, 18

Mukai, T., IL

Müller, Marcus, OU, 2I

Musolino, M., 25

Myllys, P., 2A

Najda, Stephen P., 1A

Nakamura, Shuji, $1 \mathrm{U}$

Nishitani, T., OT

Ogawa, T., $1 \mathrm{~L}$

Oh, Chan-Hyoung, $2 \mathrm{H}$

Ohtsuka, T., $1 \mathrm{~L}$

Oksanen, Jani, 1S, 2A

Okur, Serdal, 0J, 2I, 2N, 2O, 2P, 2U

Özgür, Ümit, 0J, 2I, 2J, 2N, 2O, 2P, 2U

Patrick, Erin, 12
Pearton, S. J., 12

Perlin, Piotr, $1 \mathrm{~A}$

Posthuma, Niels, 11

Rampazzo, F., 14

Rass, Jens, $1 \mathrm{~K}$

Reich, Christoph, 1K, IP

Ren, F., 12

Reshchikov, M. A., OL

Riechert, H., 25

Ristic, Jelena, 18

Riuttanen, L., 2A

Rosales, Daniel, OJ, OK, OZ

Rossetto, I., 14

Rossow, U., 1R

Sadi, Toufik, is

Scarparo, L., 25

Sellés, Julien, $0 Z$

Shatalov, Max, $1 \mathrm{M}$

Shen, YU-Jiun, $1 Z$

Shields, Philip A., IV

Shim, Jong-In, $2 \mathrm{H}$

Shin, Dong-Soo, $2 \mathrm{H}$

Shur, Michael, $1 \mathrm{M}$

Smalc-Koziorowska, Julita, IA

Sochacki, T., OF

Speck, James S., IU

Stanczyk, Szymon, 1 A

Stocco, A., 14

Stoelmacker, Christoph, $1 \mathrm{~K}$

Stoffels, Steve, 11

Stojetz, Bernhard, 18

Strauss, Uwe, 18

Su, Ying-Yong, 12

Suhir, Ephraim, OP

Suihkonen, S., 2A

Sun, Wenhong, $1 \mathrm{M}$

Suski, Tadek, $1 \mathrm{~A}$

Svensk, O., 2A

Tabuchi, M., OT

Tahraoui, A., 25

Targowski, Grzegorz, 1A

Tautz, Soenke, 18

Teke, Ali, $2 \mathrm{U}$

Thi Ngo, Huong, OK

Torres-Pardo, Almudena, OU

Tsai, Chang-Yu, $1 Z$

Tsai, Chi-Tsung, OE

Tsai, Tsung-Yen, OE

Tseng, Ming-Chun, OM

Tulkki, Jukka, is

Ueda, Daisuke, 10

Usikov, A., OL

Valvin, Pierre, OK

van der Meulen, Herko P., OU

Van Hove, Marleen, 11

Vasara, T., 2A

Veit, Peter, OU

Vennéguès, Philippe, $0 Z$

Vierheilig, Clemens, 18

Walter, Christoph, 18 
Wang, Q., 1D

Watson, Scott, 1A

Wellekens, Dirk, 11

Wernicke, Tim, 1K, IP

Weyers, Markus, 1K, $1 \mathrm{P}$

Weyher, J. L., OF

Willems, Maarten, 11

Wisniewski, Przemek, 1 A

Wu, Tian-Li, 11

Wuerfl, J., 14

WUU, Dong-Sing, OE, OM

Yamada, H., $1 \mathrm{~L}$

Yang, Jinwei, $1 M$

Yeh, J. H., $1 \mathrm{Z}$

You, Shuzhen, 11

Zanoni, E., 14, 25

Zhang, Fan, $0 \mathrm{~J}, 2 \mathrm{I}, 2 \mathrm{~J}, 2 \mathrm{~N}, 2 \mathrm{O}, 2 \mathrm{P}, 2 \mathrm{U}$

Zhao, S., 1D

Zhao, Yuji, IU

Zheng, Dong-Guang, $2 \mathrm{H}$

Proc. of SPIE Vol. $9363936301-9$

Downloaded From: https://www.spiedigitallibrary.org/conference-proceedings-of-spie on 26 Apr 2023 Terms of Use: https://www.spiedigitallibrary.org/terms-of-use 
Proc. of SPIE Vol. $9363936301-10$

Downloaded From: https://www.spiedigitallibrary.org/conference-proceedings-of-spie on 26 Apr 2023 Terms of Use: https://www.spiedigitallibrary.org/terms-of-use 


\section{Conference Committee}

Symposium Chairs

David L. Andrews, University of East Anglia (United Kingdom)

Alexei L. Glebov, OptiGrate Corporation (United States)

Symposium Co-chairs

Jean-Emmanuel Broquin, IMEP-LAHC (France)

Shibin Jiang, AdValue Photonics, Inc. (United States)

Program Track Chair

James G. Grote, Air Force Research Laboratory (United States)

Conference Chairs

Jen-Inn Chyi, National Central University (Taiwan)

Hiroshi Fujioka, The University of Tokyo (Japan)

Hadis Morkoç, Virginia Commonwealth University (United States)

Conference Co-chairs

Yasushi Nanishi, Ritsumeikan University (Japan)

Joachim Piprek, NUSOD Institute LLC (United States)

Ulrich T. Schwarz, IMTEK, Universität Freiburg (Germany)

Jong-In Shim, Hanyang University (Korea, Republic of)

Conference Program Committee

Hiroshi Amano, Nagoya University (Japan)

Michal Bockowski, Institute of High Pressure Physics (Poland)

Enrique Calleja, Universidad Politécnica de Madrid (Spain)

Shigefusa F. Chichibu, Tohoku University (Japan)

Bernard Gil, Université Montpellier 2 (France)

Nicolas Grandjean, Ecole Polytechnique Fédérale de Lausanne

(Switzerland)

Hideki Hirayama, RIKEN (Japan)

Ray-Hua Horng, National Chung Hsing University (Taiwan)

Stacia Keller, University of California, Santa Barbara (United States)

Michael Kneissl, Technische Universität Berlin (Germany)

Hao-Chung Kuo, National Chiao Tung University (Taiwan)

Masaaki Kuzuhara, University of Fukui (Japan)

Koh Matsumoto, Taiyo Nippon Sanso Corporation (Japan) 
Hideto Miyake, Mie University (Japan)

Eva Monroy, CEA Grenoble (France)

Yong-Tae Moon, LG Electronics Inc. (Korea, Republic of)

Ki-Bum Nam, Seoul Semiconductor (Korea, Republic of)

Ümit Özgür, Virginia Commonwealth University (United States)

Tae-Yeon Seong, Korea University (Korea, Republic of)

Chih-Chung Yang, National Taiwan University (Taiwan)

Euijoon Yoon, Seoul National University (Korea, Republic of)

Enrico Zanoni, Università degli Studi di Padova (Italy)

\section{Session Chairs}

1 Growth I

Ümit Özgür, Virginia Commonwealth University (United States)

2 Growth II

Michal Bockowski, TopGaN sp. z o.o. (Poland)

3 Growth III

Yasushi Nanishi, Ritsumeikan University (Japan)

4 Material Characterization I

Bernd Witzigmann, Universität Kassel (Germany)

5 Material Characterization II

Tadek Suski, Institute of High Pressure Physics (Poland)

6 Nanostructures and Devices I

Enrique Calleja, Universidad Politécnica de Madrid (Spain)

7 Nanostructures and Devices II

Joachim Piprek, NUSOD Institute LLC (United States)

8 Electron Devices I

Jen-Inn Chyi, National Central University (Taiwan)

9 Electron Devices II

Russell D. Dupuis, Georgia Institute of Technology (United States)

10 Laser Diodes I

Ulrich T. Schwarz, IMTEK, Universität Freiburg (Germany)

11 Laser Diodes II

Piotr Perlin, Institute of High Pressure Physics (Poland)

12 LEDs I

Jong-In Shim, Hanyang University (Korea, Republic of) 
13 LEDs II

Leo J. Schowalter, Crystal IS, Inc. (United States)

14 LEDs III

Hiroshi Fujioka, The University of Tokyo (Japan)

15 LEDs IV

Jen-Inn Chyi, National Central University (Taiwan)

Proc. of SPIE Vol. $9363936301-13$

Downloaded From: https://www.spiedigitallibrary.org/conference-proceedings-of-spie on 26 Apr 2023 Terms of Use: https://www.spiedigitallibrary.org/terms-of-use 
Proc. of SPIE Vol. $9363936301-14$

Downloaded From: https://www.spiedigitallibrary.org/conference-proceedings-of-spie on 26 Apr 2023 Terms of Use: https://www.spiedigitallibrary.org/terms-of-use 